\title{
Effects of Prophylactic Antibiotic Administration and Antibiotic Timing on Culture Results and Clinical Outcomes of Pediatric Musculoskeletal Infection: A Protocol for A Randomized, Controlled, Clinical Trial
}

\section{Yongjie Xia \\ Shenzhen Children's Hospital \\ Chao Deng \\ Shenzhen Children's Hospital \\ Yibiao Zhou \\ Shenzhen Children's Hospital \\ Dechao Wu \\ Shenzhen Children's Hospital \\ Zhiyong Liu \\ Shenzhen Children's Hospital \\ Liangfu Xie \\ Shenzhen Children's Hospital \\ Bing $\mathrm{E}$ \\ Shenzhen Children's Hospital \\ Jingming Han \\ Shenzhen Children's Hospital}

Chao You ( $\nabla$ youchao1978@sina.com )

Shenzhen Children's Hospital https://orcid.org/0000-0002-4782-3085

Study protocol

Keywords: pediatric musculoskeletal infection, prophylactic antibiotic administration, antibiotic timing, culture

Posted Date: January 23rd, 2021

DOI: https://doi.org/10.21203/rs.3.rs-151741/v1 
License: (c) (i) This work is licensed under a Creative Commons Attribution 4.0 International License. Read Full License 


\section{Abstract}

Background: Musculoskeletal infection (MSI) is a common cause of morbidity among the pediatric population. Some clinicians recommend withholding prophylactic antibiotics until culture collection with an aim to improve the culture sensitivity. However, a recent retrospective study reported that prophylactic antibiotic administration did not affect culture sensitivities in either disseminated or local MSI in pediatric population, which is surprising. Therefore, the aim of this study is to investigate the effects of prophylactic antibiotic administration and the timing of antibiotic administration on culture sensitivity and clinical outcomes of pediatric MSI.

Methods: A randomized, controlled, clinical trial will be carried out. Individuals aged 0 to 18 years with a diagnosis of MSI will be screened and evaluated at the Shenzhen Children's Hospital. The participants will be randomly allocated into four groups, and they will receive the antibiotic treatment at different time points, that is, 1 week, 3 days, 1 day prior to culture collection and 1 day after culture collection, respectively. The primary outcome will be culture sensitivity. In addition, the disease-related markers including white blood cell count (WBC), C-reactive protein (CRP), erythrocyte sedimentation rate, vital signs as well as the length of hospital stay will be measured or recorded accordingly. Using chi-squared tests, the rates of positive cultures will be compared between different groups. Statistical comparisons between the different patient groups regarding the confounding and outcome variables will be conducted using independent t-tests, Mann-Whitney $\mathrm{U}$ tests, chi-squared tests and Fisher's exact tests as appropriate with the significance level set to $5 \%(P<0.05)$.

Discussion: The results of this study would provide some evidence for the clinical management of pediatric MSIs with regards to the application of prophylactic antibiotics.

Trial registration: Chinese Clinical Trial Registry, ChiCTR2100041631. Registered at 1 January 2021, http://www.chictr.org.cn/hvshowproject.aspx?id=69850.

\section{Background}

Musculoskeletal infection (MSI) in pediatric population is an ongoing condition due to continuous pathogenic changes. The incidence of pediatric MSIs is approximately 2-13 every 100,000 children per year in developed countries but higher in other districts [1-4]. The MSIs consist of a wide spectrum of infections involving different musculoskeletal districts, including joint, bone, muscle and deep soft tissue. Historically, the clinical severity and presentation vary by the causative bacterium, and there has seen a significant change in osteoarticular infections pathogenesis due to emerging pathogens in the last decades $[5,6]$. Staphylococcus aureus methicillin susceptible (MSSA) has been the most frequent cause of bone and joint infections, and Kingella kingae is the most frequent cause of osteoarticular infections in pediatric patients under 4 years. The emerging pathogens have added to the complexity of pediatric MSIs. The management of MSIs requires prompt diagnosis and treatment due to the risk of local tissue 
damage and metastatic bacterial spread. Culture is the main diagnostic method to identify the causative organism which could provide hints for the following targeted antibiotic therapy.

When caring for pediatric MSI patients, the question concerning the timing of prophylactic antibiotics remain controversial at present. Traditionally, some clinicians recommend that prophylactic antibiotics should be withheld until culture collection with the aim to improve the culturing sensitivity of the causative organisms and guiding the application of antibiotics. However, in adults, conflicting studies on the effects of antibiotics on tissue culture results have been found [7-10]. Meanwhile, in other infectious diseases like sepsis [11-13], community-acquired pneumonia [14] and febrile neutropenia [15, 16], earlier antibiotic administration has shown some benefits. These conflicting findings have made it confusing when deciding whether to use prophylactic antibiotics prior to antibiotics in clinical practice. Nevertheless, a recent retrospective study surprisingly found that yields of tissue culture were not affected by antibiotic administration in either disseminated or local pediatric MSIs. In addition, another retrospective study reported that surgical culture yield in pediatric patients with acute, hematogenous, osteoarticular infection was not decreased by antibiotic administration 1 hour before surgery [17]. These results suggested that antibiotic administration delay may not be necessarily needed for better tissue culture results, which is quite a surprising suggestion [18]. Therefore, a prospective trial is needed to further evaluate the effect of antibiotic timing in pediatric MSI tissue culture results.

A randomized, controlled, clinical trial will be carried out to (1) investigate whether the administration of routine prophylactic antibiotics administration would affect the culture sensitivity during MSI treatment; (2) evaluate the effects of the antibiotic timing on the yield of cultures and clinical outcomes. This study is aimed to provide some evidence for the clinical management of pediatric MSIs with regards to the application timing of antibiotics.

\section{Methods}

\section{Ethical issues}

This protocol is a randomized controlled trial involving qualitative research, specimen (bone biopsy, fluid aspiration, etc.) collection and blood tests. The trial has received approval from the Human Research Ethics Committee of Shenzhen Children's Hospital. All the participants will sign the informed written consent before enrolled in the research.

\section{Participants}

\section{Sample size}

The software G*Power was used to generate a power analysis. Combining the results of comparable studies $[19,20]$ and theoretical considerations, the effect size was set as 0.8 , the priori test power $1-\beta$ was 0.8 , and the allocation ratio was 1 . The software generates a minimum sample size of 26 patients for each group, which is enough to investigate this effect. The assumed dropout rate is approximately 
$20 \%$. Therefore, the targeted sample size for each group should be 35 , and a total of 140 patients will meet the criteria.

\section{Inclusion criteria:}

(1) Children and adolescents with a diagnosis of MSl;

(2) Aged 0-18 years;

(3) In agreement to participate in the clinical study.

\section{Exclusion criteria:}

(1) Patients with evidence of current infections such as chronic recurrent multifocal osteomyelitis, poststreptococcal disease, necrotizing fasciitis, cellulitis or other fungal or mycobacterial infections.

(2) Patients who have recently (within 4 weeks) received any antibiotic treatment no matter related or unrelated to the MSIs.

\section{The interventions}

The enrolled patients will be stratified into disseminated or local infection groups [21]. The patients will be randomly (using computer-generated random numbers) divided into 4 groups, and will receive the antibiotic according to their allotment, that is, 1 week, 3 days, 1 day prior to culture collection and 1 day after, respectively.

\section{Clinical outcome measures}

\section{Demographic data collection}

The routine demographic data, including sex, age, classification of MSI, history of trauma, non-weightbearing at presentation, and if previously seen by medical provider will be collected and recorded.

\section{Culture}

The bacterial culture will be carried out in the Medical Center Clinical Laboratory of Shenzhen Children's Hospital. Source specimens will be collected by experienced clinicians according to the classification of MSIs, i.e. fluid aspiration for septic arthritis, subperiosteal abscess when applicable, and pyomyositis, bone biopsy for osteomyelitis.

\section{Blood test}

Markers that indicate severity of disease at presentation including blood cell count (WBC), C-reactive protein (CRP), white erythrocyte sedimentation rate will be tested accordingly.

\section{Length of hospital stay}

Length of hospital stay of each participant will be recorded. 


\section{Data and statistical analysis}

The data will be tabulated and processed using GraphPad PRISM version 7.0 and the statistical analysis will be carried out using STATA Statistical Software (College Station, TX). The statistical comparison regarding the rates of positive cultures between the different groups will be conducted by Fisher's exact tests or chi-squares tests. The confounding and outcome variables will be compared between the different groups using chi-squared tests, Fisher's exact tests or independent $t$-tests will be used as appropriate with the significance level set to $5 \%(P<0.05)$.

\section{Discussion}

The main objective of the clinical trial is to investigate whether the administration of prophylactic antibiotics will decrease the rates of positive culture of pediatric MSI treatment and to evaluate the effects of the antibiotic timing on the culture sensitivity and clinical outcomes. We hope that the results of this study would provide some evidence for the clinical management of pediatric MSIs with regards to the application of antibiotics. If the administration of prophylactic antibiotics does not decrease the culture sensitivity of pediatric MSI patients, then it is suggested that appropriate systemic antibiotics should be given to pediatric patients presenting with suspected MSIs promptly after clinical triage.

\section{Abbreviations}

CRP: C-reactive protein; MSI: musculoskeletal infection; MSSA: Staphylococcus aureus methicillin susceptible; WBC: white blood cell count.

\section{Declarations}

\section{Declaration of conflict of interest}

The authors have no conflicts of interest to disclose.

\section{Funding}

This research did not receive any specific grant from funding agencies in the public, commercial, or notfor-profit sectors.

\section{Availability of data and materials}

The datasets used and/or analyzed during the current study are available from the corresponding author on reasonable request.

\section{Authors' contributions}


YJX, CD, and CY conceived of the idea, YJX, YBZ and DCW developed the intervention, YJX, LFX, BE and CY developed the design of the trial and wrote the article. YJX and JMH did the literature research. All authors have read and approved the final manuscript.

\section{Ethics approval and consent to participate}

The study design, procedures and informed consent procedure were approved by the Human Research Ethics Committee of Shenzhen Children's Hospital. Consent to participate will be obtained from the participants.

\section{Consent for publication}

We have obtained consent to publish from the participant (or legal parent or guardian for children) to report individual patient data.

\section{References}

1. Gafur OA, Copley LA, Hollmig ST, Browne RH, Thornton LA, Crawford SE. The impact of the current epidemiology of pediatric musculoskeletal infection on evaluation and treatment guidelines. $J$ Pediatr Orthop. 2008;28:777-85.

2. Riise ØR, Kirkhus E, Handeland KS, Flatø B, Reiseter T, Cvancarova M, et al. Childhood osteomyelitisincidence and differentiation from other acute onset musculoskeletal features in a population-based study. BMC Pediatr. 2008;8:45.

3. Dartnell J, Ramachandran M, Katchburian M. Haematogenous acute and subacute paediatric osteomyelitis: a systematic review of the literature. J Bone Joint Surg Br. 2012;94:584-95.

4. Rossaak M, Pitto RP. Osteomyelitis in Polynesian children. Int Orthop. 2005;29:55-8.

5. Dodwell ER. Osteomyelitis and septic arthritis in children: current concepts. Curr Opin Pediatr. 2013;25:58-63.

6. Arnold SR, Elias D, Buckingham SC, Thomas ED, Novais E, Arkader A, et al. Changing patterns of acute hematogenous osteomyelitis and septic arthritis: emergence of community-associated methicillin-resistant Staphylococcus aureus. J Pediatr Orthop. 2006;26:703-8.

7. Ghanem E, Parvizi J, Clohisy J, Burnett S, Sharkey PF, Barrack R. Perioperative antibiotics should not be withheld in proven cases of periprosthetic infection. Clin Orthop Relat Res. 2007;461:44-7.

8. Choi HR, Kwon YM, Freiberg AA, Nelson SB, Malchau H. Periprosthetic joint infection with negative culture results: clinical characteristics and treatment outcome. J Arthroplasty. 2013;28:899-903.

9. Al-Mayahi M, Cian A, Lipsky BA, Suvà D, Müller C, Landelle C, Miozzari HH, et al. Administration of antibiotic agents before intraoperative sampling in orthopedic infections alters culture results. $J$ Infect. 2015;71:518-25.

10. Shahi A, Deirmengian C, Higuera C, Chen A, Restrepo C, Zmistowski B, et al. Premature therapeutic antimicrobial treatments can compromise the diagnosis of late periprosthetic joint infection. Clin 
Orthop Relat Res. 2015;473:2244-9.

11. Ferrer R, Martin-Loeches I, Phillips G, Osborn TM, Townsend S, Dellinger RP, et al. Empiric antibiotic treatment reduces mortality in severe sepsis and septic shock from the first hour: results from a guideline-based performance improvement program. Crit Care Med. 2014;42:1749-55.

12. Gaieski DF, Mikkelsen ME, Band RA, Pines JM, Massone R, Furia FF, et al. Impact of time to antibiotics on survival in patients with severe sepsis or septic shock in whom early goal-directed therapy was initiated in the emergency department. Crit Care Med. 2010;38:1045-53.

13. Kumar A, Roberts D, Wood KE, Light B, Parrillo JE, Sharma S, et al. Duration of hypotension before initiation of effective antimicrobial therapy is the critical determinant of survival in human septic shock. Crit Care Med. 2006;34:1589-96.

14. Huang JQ, Hooper PM, Marrie TJ. Factors associated with length of stay in hospital for suspected community-acquired pneumonia. Can Respir J. 2006;13:317-24.

15. Perron T, Emara M, Ahmed S. Time to antibiotics and outcomes in cancer patients with febrile neutropenia. BMC Health Serv Res. 2014;14:162.

16. Fletcher M, Hodgkiss H, Zhang S, Browning R, Hadden C, Hoffman T, et al. Prompt administration of antibiotics is associated with improved outcomes in febrile neutropenia in children with cancer. Pediatr Blood Cancer. 2013;60:1299-306.

17. van der Merwe M, Rooks K, Crawford H, Frampton CMA, Boyle MJ. The effect of antibiotic timing on culture yield in paediatric osteoarticular infection. J Child Orthop. 2019;13:114-9.

18. Benvenuti MA, An TJ, Mignemi ME, Martus JE, Thomsen IP, Schoenecker JG. Effects of Antibiotic Timing on Culture Results and Clinical Outcomes in Pediatric Musculoskeletal Infection. J Pediatr Orthop. 2019;39:158-62.

19. Burnett RS, Aggarwal A, Givens SA, McClure JT, Morgan PM, Barrack RL. Prophylactic antibiotics do not affect cultures in the treatment of an infected TKA: a prospective trial. Clin Orthop Relat Res. 2010;468:127-34.

20. Dlamini LD, Sekikubo M, Tumukunde J, Kojjo C, Ocen D, Wabule A, et al. Antibiotic prophylaxis for caesarean section at a Ugandan hospital: a randomised clinical trial evaluating the effect of administration time on the incidence of postoperative infections. BMC Pregnancy Childbirth. 2015;15:91.

21. Mignemi ME, Benvenuti MA, An TJ, Martus JE, Mencio GA, Lovejoy SA, et al. A Novel Classification System Based on Dissemination of Musculoskeletal Infection is Predictive of Hospital Outcomes. J Pediatr Orthop. 2018;38:279-86. 